\title{
Mixed kinematic and dynamic sideslip angle observer for accurate control of fast off-road mobile robots
}

\author{
Roland Lenain \\ Cemagref \\ 24 avenue des Landais \\ 63172 Aubière, France \\ roland.lenain@cemagref.fr \\ Christophe Cariou \\ Cemagref \\ 24 avenue des Landais \\ 63172 Aubière, France \\ christophe.cariou@cemagref .fr
}

\author{
Benoit Thuilot \\ Lasmea \\ 24 avenue des Landais \\ 63177 Aubière, France \\ thuilot@lasmea.univ-bpclermont.fr \\ Philippe Martinet \\ Lasmea \\ 24 avenue des Landais \\ 63177 Aubière, France \\ martinet@lasmea.univ-bpclermont.fr
}

\begin{abstract}
Automation in outdoor applications (farming, surveillance, military activities, etc.) requires highly accurate control of mobile robots, at high speed, although they are moving on low grip terrain. To meet such expectations, advanced control laws accounting for natural ground specificities (mainly sliding effects) must be derived. In previous work, adaptive and predictive control algorithms, based on an extended kinematic representation, have been proposed. Satisfactory experimental results have been reported (accurate to within $\pm 10 \mathrm{~cm}$, whatever grip conditions), but at limited velocity (below $3 m . s^{-1}$ ). Nevertheless, simulations reveal that control accuracy is decreased when vehicle speed is increased (up to $10 \mathrm{~m} . \mathrm{s}^{-1}$ ). In particular, oscillations are observed at curva-
\end{abstract}


ture transition.

This drawback is due to delays in sideslip angle estimation, unavoidable at high speed since only an extended kinematic representation was used. In this paper, a mixed backstepping kinematic and dynamic observer is designed to improve observation of these variables: the slow-varying data are still estimated from a kinematic representation, which is then injected into a dynamic observer to supply reactive and reliable sliding variable (namely sideslip angle) estimation, without increasing the noise level. The algorithm is evaluated via advanced simulations (coupling Adams and Matlab software) investigating high speed capabilities. Actual experiments at lower speed (experimental platform maximum velocity), demonstrate the benefits of the proposed approach.

\section{Introduction}

The development of autonomous mobile robots acting at high speed in a natural environment constitutes an important issue, considering the numerous fields of application (see (Siegwart and Nourbakhsh, 2004)). From transportation to agricultural operations (Bruinsma, 2003) (not to mention exploration, surveillance, military activities, etc.), the benefits brought by such robots may indeed improve both the safety and the efficiency of the required tasks. As a result, the robotic research related to such a topic is the object of increasing interest (as highlighted in (Buehler et al., 2007)), bringing together many research areas, such as sensing, planning or control.

This latter area has been addressed in the work of numerous researchers, but only a few have specifically considered fast off-road mobile robots. Control of wheeled robots has indeed been studied in depth for on-road vehicles, indoor robots or urban transportation systems (see for instance (Micaelli and Samson, 1993)). In such a context, simplifying assumptions (mainly pure rolling and non-sliding at tire/soil contact point) can be introduced, and control law design can then be based on kinematic models. Alas, in off-road conditions, the complexity 
of the phenomena encountered and their variability do not permit reliance on such hypotheses. As a consequence, direct transfer of this work to the off-road context, and especially at high speed, is impossible without a significant loss in terms of precision. Dynamic vehicle representations (general dynamic vehicle models, as in (Unyelioglu et al., 1997), or partial ones focused on tire/ground contact forces (e.g. (Ellouze and d'Andréa-Novel, 2000)), steering actuator dynamics (e.g. (Tai et al., 2004)), etc.) could account accurately for fast and complex robot behaviors. However, they involve numerous parameters, and are therefore tractable for control purposes only when vehicles move in structured and non-varying environments, such as asphalted roads. As an alternative, some approaches consider the specific phenomena encountered in a natural environment (mainly due to wheel skidding) as perturbations in the kinematic model to be rejected (see (Wang and Low, 2007) for instance). Some robust control techniques are then designed to compensate for such perturbations (as investigated in (Eaton et al., 2008), (Hao et al., 2005), or (Lucet et al., 2008)). However, these approaches tend to be conservative, even at limited speed, and oscillating behavior can be recorded when significant sliding occurs.

In previous work, another approach was used: additional variables, consistent with a dynamic vehicle representation, are estimated on-line and then incorporated into what is called an extended kinematic model, from which control laws are designed. As detailed in (Lenain et al., 2007) for agricultural vehicles, or in (Cariou et al., 2008) for more generic off-road mobile robots, these observer-based adaptive and predictive control laws are relevant in an off-road context: at the velocity range considered in these applications (up to $10 \mathrm{~km} . \mathrm{h}^{-1}$ ), high accuracy can be ensured whatever the grip conditions and terrain interaction encountered. However, if higher speeds are expected (up to $40 \mathrm{~km} \cdot \mathrm{h}^{-1}$ in the near future), the efficiency of the proposed approach decreases: since the observer is designed from an extended kinematic representation, a delay in the estimation of the sliding variables (homogeneous with sideslip angles) progressively appears and is particularly disadvantageous from a control point of view (oscillations can then be noticed, especially at curvature transitions). In order to allow a significant increase in vehicle velocity, dynamic effects have to be taken into account in the 
sliding observer, without however introducing parameters that are hardly measurable.

This paper addresses the design of such an observer. It can be broken down into two steps. The first aims at adapting on-line the cornering stiffnesses (i.e. estimation of the variations in grip conditions), to be injected into the second step, dedicated to sideslip angle observation from a dynamic vehicle model. The previous observer (based on an extended kinematic vehicle model) is still used in parallel, in order that no preliminary knowledge with respect to the terrain may be needed. Such an approach dispenses with any need of on-line direct measurement of grip conditions (hardly feasible at reasonable cost), when the use of a dynamic model considerably reduces the delay in sliding variable estimation. As a result, the accuracy of mobile robot motion in off-road conditions can be preserved, even at high speed.

The paper is organized as follows: in the first part, previous work is recalled, including extended kinematic modeling, preliminary sideslip angle estimation, and design of the adaptive and predictive control law, still used to demonstrate the capabilities of the approach. In the second part, the proposed observer is introduced: dynamic modeling, cornering stiffness adaptation, and the dynamic sideslip angle observer itself are successively presented. Advanced simulations, as well as experimental results, are then proposed to show the efficiency of the algorithm at high speed.

\section{Previous work}

\subsection{Kinematic modeling with sliding taken into account}

Usually, vehicle motion is derived from a kinematic representation based on the assumption of rolling without sliding at the tire/ground contact point (see for instance (Samson, 1995)). If such a hypothesis is relevant in the urban vehicle context, its direct transposition to allterrain vehicle leads to inaccurate results. Consequently, low grip conditions have to be 
properly modeled. In this paper, it is proposed to incorporate variables representative of sliding phenomena, namely tire sideslip angles, into the kinematic model. These variables (used in dynamic vehicle models to derive contact forces, see (Bakker et al., 1987) or (Ryu et al., 2008)) denote the difference between wheel plane orientation and actual speed vector direction.

Since in a kinematic framework, mobile robots are usually described as a bicycle (see Figure 1), two tire sideslip angles $\beta_{F}$ and $\beta_{R}$ are considered, respectively for the front and rear axle. It is assumed that they are entirely representative of sliding effects. Moreover, as path tracking control in off-road conditions is here addressed, vehicle modeling is derived with respect to the path to be followed (denoted $\Gamma$ ). The notations used in the sequel (see also Figure 1) are:

- $F$ and $R$ are respectively the centers of the front and rear axles, where the virtual wheels of the bicycle model are located. $R$ is the point to be controlled.

- $v$ is the vehicle linear velocity at point $R$, assumed to be strictly positive and manually controlled, and $v_{F}$ denotes the linear velocity at the center $F$ of the front wheel.

- $\delta_{F}$ is the measured front steering angle obtained thanks to an angular sensor. It constitutes the control variable and its measure permits both to feed observer algorithms and to ensure the low level closed loop control of the steering actuator.

- $\beta_{F}$ and $\beta_{R}$ are the front and rear tire sideslip angles.

- $M$ is the point on the reference path $\Gamma$, which is the closest to $R$. $M$ is assumed to be unique.

- $s$ is the curvilinear abscissa of point $M$ along $\Gamma$.

- $A(s)$ denotes the curvature center of the path $\Gamma$ at point $M$, so that $c(s)$ is the curvature of $\Gamma$ at that point.

- $y$ is the vehicle lateral deviation at point $R$ with respect to $\Gamma$.

- $\theta$ is the orientation of vehicle centerline with respect to an absolute frame $\left[O, X_{O}, Y_{O}\right)$. 
- $\theta_{\Gamma}(s)$ is the orientation of the tangent to $\Gamma$ at point $M$ with respect to $\left[O, X_{O}, Y_{O}\right)$.

- $\tilde{\theta}=\theta-\theta_{\Gamma}(s)$ is the vehicle angular deviation with respect to $\Gamma$.

- $L$ is the vehicle wheelbase.

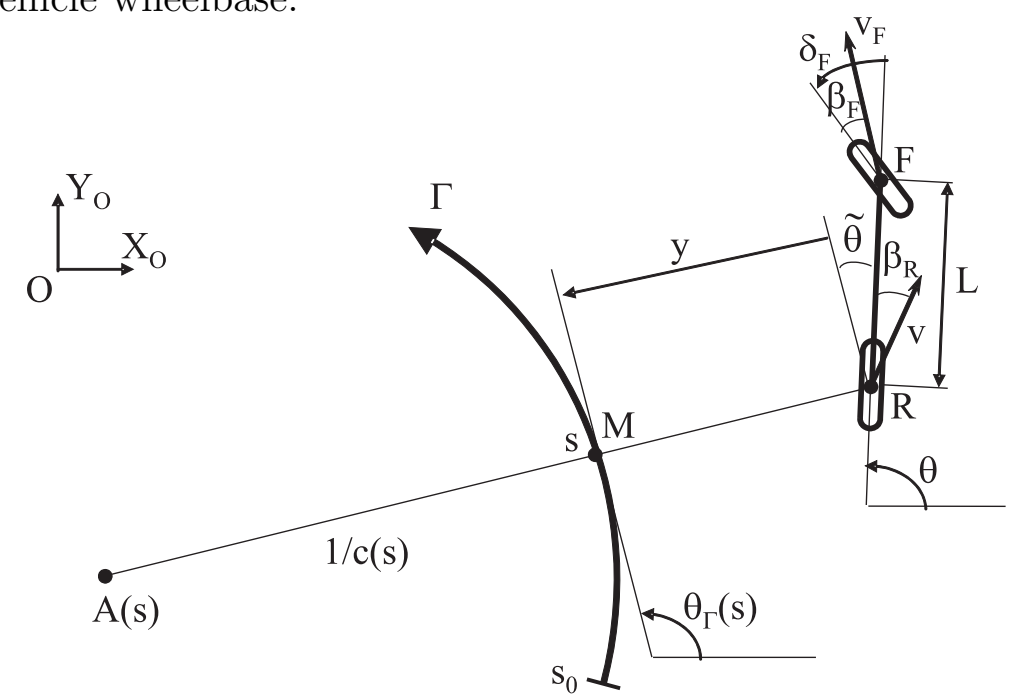

Figure 1: Path tracking parameters

In this configuration, according to sign conventions, $y, \tilde{\theta}$, $\beta_{R}, \beta_{F}$ have negative values, when $\delta_{F}$ has a positive value

It can be established (see (Samson, 1995) for model derivation in the non-sliding case and (Lenain et al., 2006) for the integration of the sideslip angles) that:

$$
\left\{\begin{aligned}
\dot{s} & =v \frac{\cos \left(\tilde{\theta}+\beta_{R}\right)}{1-c(s) y} \\
\dot{y} & =v \sin \left(\tilde{\theta}+\beta_{R}\right) \\
\dot{\tilde{\theta}} & =v\left[\cos \left(\beta_{R}\right) \lambda_{1}-\lambda_{2}\right]
\end{aligned}\right.
$$

with: $\lambda_{1}=\frac{\tan \left(\delta_{F}+\beta_{F}\right)-\tan \left(\beta_{R}\right)}{L}, \lambda_{2}=\frac{c(s) \cos \left(\tilde{\theta}+\beta_{R}\right)}{1-c(s) y}$

It can be observed that this model becomes singular when $y=\frac{1}{c(s)}$, i.e. when $R$ is superposed with $A(s)$. This problem is not encountered in practice since, on the one hand actual path curvatures are quite small, and on the other hand, the vehicle remains close to $\Gamma$ when properly initialized. The lateral deviation is thus always smaller than the radius of curvature 
of $\Gamma$.

\subsection{Preliminary sideslip angle estimation}

In order to derive control laws from the model (1), sideslip angles have to be available. The current robot state, as well as the modifications in terrain configuration (i.e. grip conditions), have a significant impact on both these variables, particularly in an off-road context. As a result, $\beta_{F}$ and $\beta_{R}$ must be estimated on-line.

To this end, it was proposed in (Lenain et al., 2007) to apply observer theory to the model (1). Since two variables have to be estimated, two output variables were associated with the model (1), namely $X_{o b s}=(y, \tilde{\theta})_{o b s}$. The two sideslip angles were then seen as control variables, and a control law was proposed for these variables in order to ensure the convergence of $X_{o b s}$ to the measured variables $X_{m e s}=(y, \tilde{\theta})_{m e s}$.

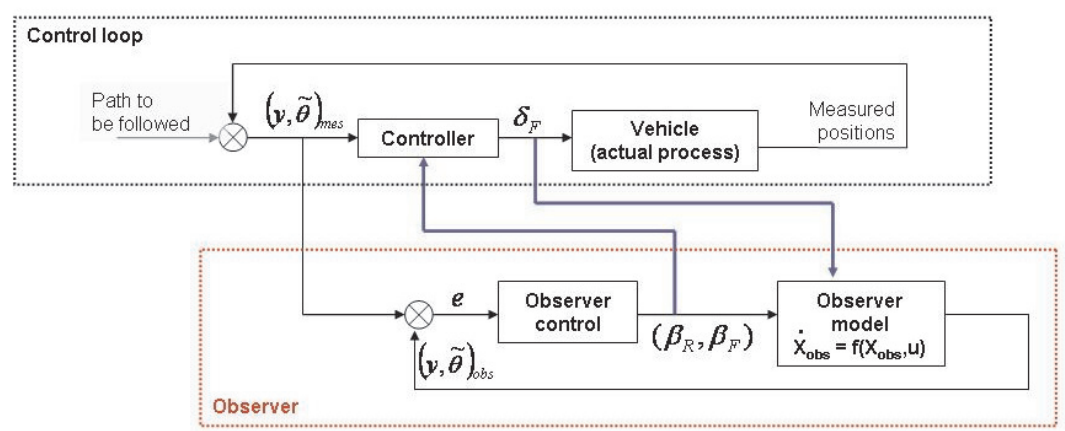

Figure 2: Diagram of the preliminary extended kinematic observer

More precisely, the extended model (1), when its first equation is dropped, can be rewritten as the non-linear state representation (2), where $\delta_{F}$ is considered as a known parameter (supplied on-line by the sensor of the steering device), and $u=\left(u_{1}, u_{2}\right)=\left(\beta_{F}, \beta_{R}\right)$ represents the variables to be controlled:

$$
\dot{X}_{o b s}=f\left(X_{o b s}, \delta_{F}, u\right)=\left(\begin{array}{l}
v \sin \left(\tilde{\theta}_{o b s}+u_{2}\right) \\
v\left[\frac{\cos \left(u_{2}\right)\left[\tan \left(\delta_{F}+u_{1}\right)-\tan \left(u_{2}\right)\right]}{L}-\frac{c(s) \cos \left(\tilde{\theta}_{o b s}+u_{2}\right)}{1-c(s) y_{o b s}}\right]
\end{array}\right)
$$

As sideslip angles do not exceed a few degrees in practice, this state equation can be linearized 
with respect to the control vector $u$ in the vicinity of zero (i.e. no sliding). It leads to:

$$
\dot{X}_{o b s}=f\left(X_{o b s}, \delta_{F}, 0\right)+B\left(X_{o b s}, \delta_{F}\right) u
$$

with $B(.,$.$) denoting the derivative of f$ with respect to $u$, evaluated at $u=(0,0)$ :

$$
B\left(X_{o b s}, \delta_{F}\right)=\left[\begin{array}{cc}
0 & v \cos \left(\tilde{\theta}_{o b s}\right) \\
\frac{v}{L \cos ^{2} \delta_{F}} & v \frac{c(s) \sin \left(\tilde{\theta}_{o b s}\right)}{1-c(s) y_{o b s}}-\frac{v}{L}
\end{array}\right]
$$

Matrix $B$ is invertible provided $\tilde{\theta}_{o b s} \neq \frac{\pi}{2}, \frac{3 \pi}{2}$ and $v \neq 0$. Such conditions are met in practical path-following conditions. Equation (3) can then be exactly linearized by imposing:

$$
u=B\left(X_{o b s}, \delta_{F}\right)^{-1}\left(m-f\left(X_{o b s}, \delta_{F}, 0\right)\right)
$$

with $m$ denoting auxiliary control variables.

Finally, let us introduce the observation error $e=X_{o b s}-X_{m e s}$. Sliding parameters can then be relevantly estimated by injecting $m=G e+\dot{X}_{m e s}$ into (5), since the error dynamic is thus given by $\dot{e}=G e$. The $(2 \times 2)$ matrix $G$, to be chosen as a Hurwitz matrix, constitutes the observer gain imposing the settling times for the observed state. In the sequel, $G$ is chosen as a diagonal matrix, in order to decouple the convergence of the two observed variables.

The term $\dot{X}_{m e s}$ is evaluated via numerical derivation, and therefore has to be slightly filtered in order to ensure its relevance despite the measurement noise. Since convergence of the observed state with the measured one has then been achieved, $u$ can be regarded as a relevant estimation of the sideslip angles.

\subsection{Path tracking control algorithm}

Since all the variables in the model (1) are now available, control law design can be addressed. As this paper is mainly focused on sideslip angle observer design, the derivation of the path tracking control law (the initial motivation for investigating sideslip angle reconstruction) is only sketched below. Further details can be found in (Lenain et al., 2006). 


\subsubsection{Adaptive control algorithm}

The model (1), like any mobile robot kinematic model, can be turned into an exact linear form, named a chained system form (see (Samson, 1995)), via state and control transformations and a time-scale change (derivation with respect to curvilinear abscissa instead of derivation with respect to time). Relying on this linear form, a classical PD control can then be designed to ensure the convergence of the actual lateral deviation to zero, while the angular deviation compensates the rear tire sideslip angle. The reverse transformations finally provide the non-linear expression (6) for the steering control law for path tracking:

$$
\delta_{F}=\arctan \left(\tan \left(\beta_{R}\right)+\frac{L}{\cos \left(\beta_{R}\right)}\left(\frac{c(s) \cos \tilde{\theta}_{2}}{\alpha}+\frac{A \cos ^{3} \tilde{\theta}_{2}}{\alpha^{2}}\right)\right)-\beta_{F}
$$

with:

$$
\left\{\begin{aligned}
\tilde{\theta}_{2} & =\tilde{\theta}+\beta_{R} \\
\alpha & =1-c(s) y \\
A & =-K_{p} y-K_{d} \alpha \tan \tilde{\theta}_{2}+c(s) \alpha \tan ^{2} \tilde{\theta}_{2}
\end{aligned}\right.
$$

In a natural environment, grip conditions are always changing. The adapted sideslip angle variables injected into the control law (6) are able to account for such fast modifications more efficiently than standard approaches attempting to reject them asymptotically (such as introducing an integral term into path tracking control laws designed for mobile robots moving without sliding). More accurate tracking performance can then be achieved, see (Lenain et al., 2006).

\subsubsection{Predictive algorithm}

Nevertheless, when relying on the previous adaptive control law, transient overshoots in lateral deviation can still be observed when the vehicle enters a curve. These overshoots are mainly due to delays induced by the steering actuator, whose dynamic is not described in the model (1). The steering angle value obtained by control law is indeed sent to a low level control loop with its own delay and settling time (0.1s and 0.8 s respectively) and servoing is ensured thanks to an absolute angle encoder. In order to account for such delays and 
then prevent transient overshoots in path tracking, a predictive curvature servoing has been proposed in (Lenain et al., 2007), with the aim of sending the steering set point to the front wheel actuator in advance. In order to impose such a behavior, the control law (6) is split into two terms:

$$
\begin{aligned}
\delta_{F} & =\delta_{\text {Traj }}+\delta_{\text {Deviation }} \\
\delta_{\text {Traj }} & =\arctan \left(g_{1}\right) \\
\delta_{\text {Deviation }} & =\arctan \left(\frac{g_{2}}{1+g_{1} g_{2}+g_{1}^{2}}\right)-\beta_{F}
\end{aligned}
$$

where:

$$
\begin{aligned}
& g_{1}=\frac{L}{\cos \left(\beta_{R}\right)} c(s) \frac{\cos \tilde{\theta}_{2}}{\alpha} \\
& g_{2}=\frac{L}{\cos \left(\beta_{R}\right)} A \frac{\cos ^{3} \tilde{\theta}_{2}}{\alpha^{2}}+\tan \left(\beta_{R}\right)
\end{aligned}
$$

This separation leads to a control expression split into two main tasks:

- $\delta_{T r a j}$ : non-null term when deviations and sliding are equal to zero. This term mainly depends on reference path curvature. It will be used to design the prediction algorithm.

- $\delta_{\text {Deviation }}$ : null term when deviations and sliding are equal to zero. This term mainly depends on sliding parameters and deviations $\left(y, \tilde{\theta}_{2}\right)$ and ensures their convergence to 0 .

As sliding conditions are unpredictable, the prediction algorithm can only be applied to $\delta_{\text {Traj }}$. From the knowledge of the reference path, the future path curvature value at time $t+H$ is inferred and used to compute the objective $\delta^{O b j}$ to be reached by $\delta_{\text {Traj }}$ at time $t+H$.

Then, relying on Model Predictive Control (MPC, see (Richalet, 1993)), a control sequence for $\delta_{\text {Traj }}$ is computed with the aim of minimizing, on the time window $[t, t+H]$, the quadratic difference between a chosen shape $\delta^{R e f}$ joining $\delta^{O b j}$ at $t+H$ and the expected steering angle $\hat{\delta}^{\text {actual }}$ computed from the actuator model, see Figure 3.

The first term of this optimum control sequence, called $\delta_{\text {Traj }}^{\text {Pred }}$, is then substituted for the 


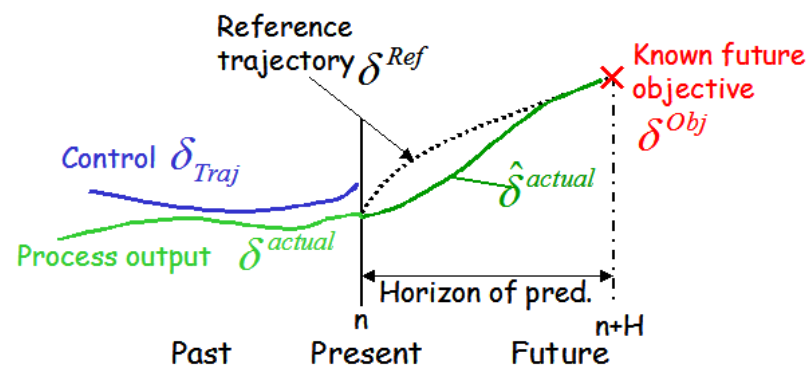

Figure 3: Notations and general description of Model Predictive Control

previous trajectory term $\delta_{\text {Traj }}$, leading to the following overall control law expression:

$$
\delta_{F}=\delta_{\text {Traj }}^{\text {Pred }}+\delta_{\text {Deviation }}
$$

Further details can be found in (Lenain et al., 2007).

\subsection{Capabilities and limitations}

As reported for example in (Lenain et al., 2007) or in (Cariou et al., 2008), such an approach ensures highly accurate path tracking (close to the localization sensor accuracy, i.e. a few centimeters when an RTK-GPS device is used), independently of the vehicle to be controlled (farm tractor, off-road mobile robot, etc.), the shape of the path to be followed, grip conditions or terrain geometry (flat or sloping field). Furthermore, the chosen representation does not demand dynamic vehicle parameters, which are not so easy to measure and are subject to change. Finally, control law performance is specified with respect to a curvilinear abscissa, so that the vehicle's behavior is independent of its velocity.

Nevertheless, these very satisfactory performances can be obtained only at quite limited velocity (below $10 \mathrm{~km} . \mathrm{h}^{-1}$, from our experimental records). At higher speed, oscillations may appear, especially at transient phases. Several reasons could explain this fact:

- Sampling frequency and sensor limitations. In previous work, the control law sampling frequency was chosen with respect to the features of the sole exteroceptive sensor used for guidance: an RTK GPS running at 10Hz. Naturally, such a choice restrains 
the maximum reachable velocity, since the Shannon theorem has to be satisfied. Nevertheless, this limitation (around $30 \mathrm{~km} . \mathrm{h}^{-1}$ ) is much higher than the velocities for which oscillations have been experimentally observed (from $15 \mathrm{~km} . \mathrm{h}^{-1}$ ). Moreover, simulation runs carried out with a $20 \mathrm{~Hz}$ sampling frequency for both positioning information and control law show similar oscillating behavior in low grip conditions.

- Kinematic representation. A pure kinematic representation cannot reflect dynamic phenomena acting at high speed (inertial effects, tire/ground contact transitions, etc.) Nevertheless, since an "extended kinematic model" accounting for skidding effects via on-line adapted sideslip angles has been considered, an accurate description of off-road mobile robot motion can be expected, as long as sideslip angles can be satisfactorily estimated.

- Observer algorithm. The proposed sideslip angle observer is indeed the main limitation at high speed. On the one hand, it disregards any dynamic phenomenon, and on the other hand its settling time must be limited in order to filter out sensor noise. As a consequence, sliding estimation is noticeably delayed and inaccurate at transient phases (even if it supplies relevant steady values). At low speed, the influence of this delay is negligible, but this is no longer true at higher speed. This can easily be checked in simulation: if simulated sideslip angles (available in simulation) are reported into control algorithms, then oscillations are no longer observed during path tracking.

As a result, preservation of high accuracy path tracking at high speed demands the integration of dynamic features into the observer algorithm, in order to decrease estimation delays and therefore improve guidance accuracy at transient phases. 


\section{Mixed kinematic and dynamic sliding estimation}

\subsection{Dynamic modeling}

In order to incorporate dynamic effects into sideslip angle estimation, a dynamic model describing mobile robot motion in a yaw frame is first designed. As previously, a bicycle vehicle is considered (see Figure 4) and the velocity is supposed to be slow-varying, so that longitudinal forces can be disregarded. With respect to the extended kinematic representation depicted in Figure 1, the additional variables and notations are:

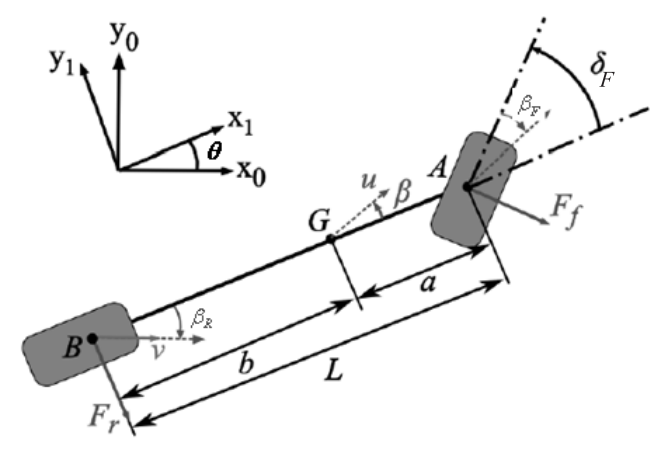

Figure 4: Dynamic bicycle model with sliding parameters.

- $G$ is the vehicle center of gravity,

- $a$ and $b$ are respectively the front and rear half-wheelbases,

- $u$ is the linear velocity at the center of gravity,

- $\beta$ is the vehicle sideslip angle,

- $F_{f}$ and $F_{r}$ are respectively the lateral forces generated at the front and rear tires.

- $m$ is the total mobile robot mass.

- $I_{z}$ is the moment of inertia about the vertical axis passing through the vehicle center of gravity (i.e. at $G$ ). 
Using these notations, a dynamic model describing robot motion in a yaw frame (see (Schofield et al., 2006) or (Ellouze and d'Andréa-Novel, 2000)) is:

$$
\left\{\begin{aligned}
\ddot{\theta} & =\frac{1}{I_{z}}\left(-a F_{f} \cos \left(\delta_{F}\right)+b F_{r}\right) \\
\dot{\beta} & =-\frac{1}{u m}\left(F_{f} \cos \left(\beta-\delta_{F}\right)+F_{r} \cos (\beta)\right)-\dot{\theta} \\
\beta_{R} & =\arctan \left(\tan \beta-\frac{b \dot{\theta}}{u \cos (\beta)}\right) \\
\beta_{F} & =\arctan \left(\tan \beta+\frac{a \dot{\theta}}{u \cos (\beta)}\right)-\delta_{F} \\
u & =v \frac{\cos \left(\beta_{R}\right)}{\cos (\beta)}
\end{aligned}\right.
$$

In order to design an observer from the model (11), lateral forces $F_{f}$ and $F_{r}$ have first to be evaluated. Numerous models are available in the literature to compute such forces (see for example (Bakker et al., 1987) or (Canudas de Wit et al., 1995)). Nevertheless, most of them link the lateral forces to tire sideslip angles in a non-linear way, as depicted by the black dashed line in Figure 5.

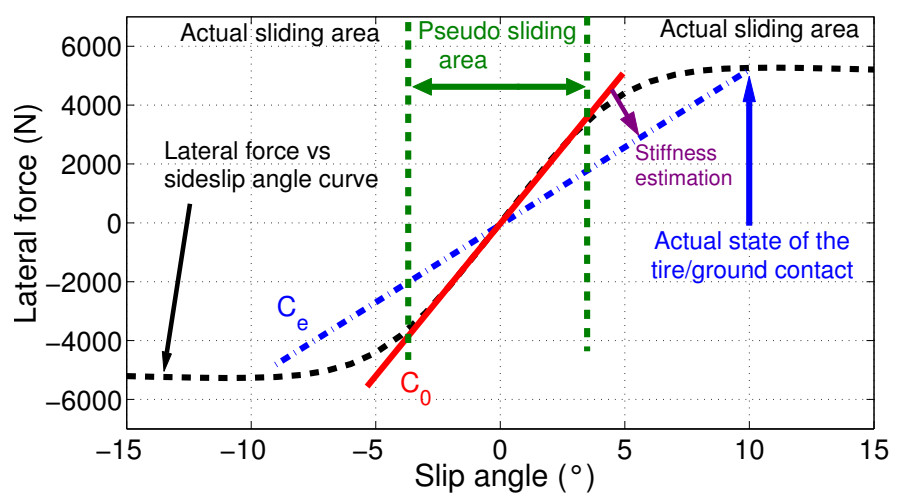

Figure 5: Non-linear tire behavior, here modeled with varying cornering stiffness

Such relations rely on numerous parameters, difficult to identify and moreover varying with the type of contact and terrain, tire load, etc. When considering all-terrain applications, these numerous parameters cannot definitely be assumed as constant during vehicle motion. As a result, in order to keep the model (11) tractable, $F_{f}$ and $F_{r}$ are here viewed as linear functions of front and rear tire sideslip angles $\left(\beta_{F}\right.$ and $\left.\beta_{R}\right)$, but relying on varying cornering stiffnesses $C_{F}$ and $C_{R}$ :

$$
\left\{\begin{array}{c}
F_{f}=C_{F}(\text { vehicle/terrain config. }) \beta_{F} \\
F_{r}=C_{R}(\text { vehicle/terrain config. }) \beta_{R}
\end{array}\right.
$$


On-line estimation of $C_{F}$ and $C_{R}$ can take into account, not only varying grip conditions, but also the non-linear parts of the tire model (as depicted with the blue dashed line in Figure 5). Estimation algorithms have been proposed in (Bouton et al., 2008) or in (Msirdi et al., 2008). An alternative one is presented in Section 3.3.

Injecting (12) into the model (11) then leads to:

$$
\left\{\begin{aligned}
\ddot{\theta} & =\frac{1}{I_{z}}\left(-a C_{F} \beta_{F} \cos \left(\delta_{F}\right)+b C_{R} \beta_{R}\right) \\
\dot{\beta} & =-\frac{1}{u m}\left(C_{F} \beta_{F} \cos \left(\beta-\delta_{F}\right)+C_{R} \beta_{R} \cos (\beta)\right)-\dot{\theta} \\
\beta_{R} & =\arctan \left(\tan \beta-\frac{b \dot{\theta}}{u \cos (\beta)}\right) \\
\beta_{F} & =\arctan \left(\tan \beta+\frac{a \dot{\theta}}{u \cos (\beta)}\right)-\delta_{F} \\
u & =\frac{v \cos \left(\beta_{R}\right)}{\cos (\beta)}
\end{aligned}\right.
$$

Considering now that, in practice, sideslip angles $\left(\beta_{F}, \beta_{R}\right.$ and $\left.\beta\right)$ are quite small and that nonlinear tire behavior has been taken into account via the adaptation of cornering stiffnesses, the model (13) can be linearized around null sideslip angles, leading to the dynamic model to be considered in the sequel:

$$
\left\{\begin{aligned}
\ddot{\theta} & =\frac{1}{I_{z}}\left(-a C_{F} \beta_{F} \cos \left(\delta_{F}\right)+b C_{R} \beta_{R}\right) \\
\dot{\beta} & =-\frac{1}{u m}\left(C_{F} \beta_{F} \cos \left(\delta_{F}\right)+C_{R} \beta_{R}\right)-\dot{\theta} \\
\beta_{R} & =\beta-\frac{b \dot{\theta}}{u} \\
\beta_{F} & =\beta+\frac{a \dot{\theta}}{u}-\delta_{F} \\
u & =v
\end{aligned}\right.
$$

\subsection{General algorithm description}

The objective is now to derive on-line estimation of tire sideslip angles $\beta_{F}$ and $\beta_{R}$ from the dynamic model (14), with the aim of eventually improving the reactivity of the overall path tracking algorithm (with respect to what was previously obtained when $\beta_{F}$ and $\beta_{R}$ were supplied by the observer (5), derived from the extended kinematic model (1)). As can be seen from the model (14), $\beta_{F}$ and $\beta_{R}$ are directly related to yaw rate $\dot{\theta}$ and vehicle sideslip 
angle $\beta$. The former variable can be measured by an on-board sensing system (either from an RTK-GPS receiver or from a low cost gyrometer), but not the latter one. Moreover, vehicle sideslip angle equation relies on cornering stiffnesses $C_{F}$ and $C_{R}$, representative of grip conditions, unknown and time-varying.

As a result, a mixed kinematic and dynamic observer is here proposed for sideslip angle estimation. Its general principle is described in Figure 6. With respect to the previous observer scheme shown in Figure 2, the control loop (top of the figure) is unchanged and still fed with the estimated front and rear tire sideslip angles. In contrast, the observation loop consists now of three successive steps, each one relying on the variable supplied by the preceding step. This approach to observer design is therefore similar, to some extent, to the celebrated backstepping control design technique. The three blocks shown in Figure 6 are described below:

- Preliminary extended kinematic observer (red dashed box). This is the observer (5). As discussed before, it can supply relevant tire sideslip angle estimation at low speed. However, when higher speeds are considered, estimation is delayed, especially at transient phases, and is no longer suitable for vehicle control. Nevertheless, it constitutes a preliminary slow-varying approximation of the targeted estimates, relevant in a steady state. It is therefore used below as an input for the other two observation steps.

- Cornering stiffness observer (green dotted box). This second step of the observation loop is concerned with on-line adaptation of cornering stiffnesses, with the aim of reflecting grip condition variations. More precisely, stiffnesses are viewed as the control variables, and a control law is designed to ensure that the observed yaw rate built from the dynamic model (14) converges with the measured rate, and that the observed vehicle sideslip angle converges with the value inferred from the observer (5). However, as mentioned above, the observer (5) supplies a delayed estimation for sideslip angles, relevant only in a steady state. Therefore, a high priority is given here, via 
gain tuning, to the convergence of the observed yaw rate (since yaw rate measurement is reliable), in order to rely mainly on the dynamic model (14). $C_{R}$ and $C_{F}$ can then reactively describe actual terrain conditions. This cornering stiffness observer is detailed in section 3.3 .

- Dynamic sideslip angle observer (blue dashed dotted box). This last step constitutes the formal sideslip angle observer, derived from standard observer theory applied to the dynamic model (14), where the cornering stiffness estimations obtained in previous step have been reported. Once more, priority is given, via gain tuning, to the convergence of the observed yaw rate, in order that sideslip angle estimation shall mainly rely on the dynamic model (14), and not on the values supplied by the preliminary observer (5). This dynamic sideslip angle observer is detailed in section 3.4.

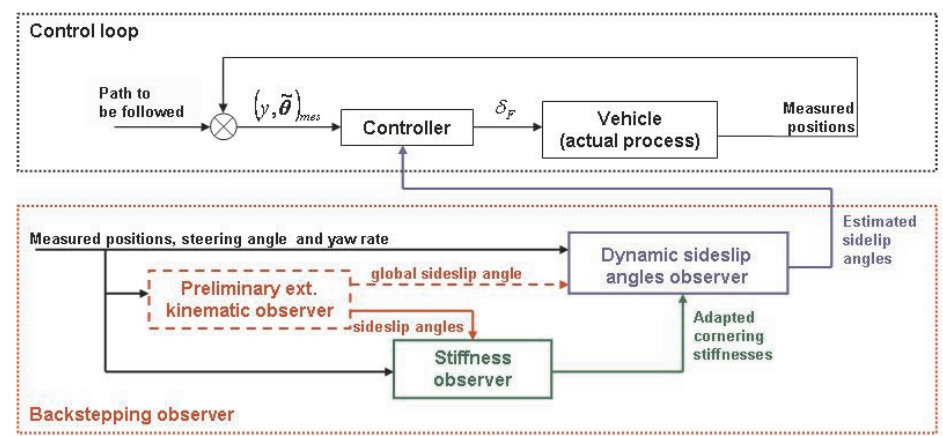

Figure 6: Scheme of the mixed kinematic and dynamic observer

\subsection{Cornering stiffness observation}

Let us denote $\bar{\beta}_{F}$ and $\bar{\beta}_{R}$, the tire sideslip angle estimations supplied by the extended kinematic observer (5). A preliminary estimation of the vehicle sideslip angle, denoted $\bar{\beta}$, can then be immediately inferred from the model (14):

$$
\bar{\beta}=\frac{b \bar{\beta}_{F}+a \bar{\beta}_{R}+b \delta_{F}}{L}
$$


A first observer model is then built from the dynamic model (14), by considering $X_{1}=$ $\left[\begin{array}{ll}\dot{\hat{\theta}}_{1} & \hat{\beta}_{1}\end{array}\right]^{T}$ as the observed state and $u=\left[\begin{array}{ll}C_{F} & C_{R}\end{array}\right]^{T}$ as the control vector. This leads to:

$$
\dot{X}_{1}=A_{1} X_{1}+B_{1} u
$$

where:

$$
A_{1}=\left[\begin{array}{cc}
0 & 0 \\
-1 & 0
\end{array}\right], \quad B_{1}=\left[\begin{array}{cc}
-\frac{a \bar{\beta}_{F} \cos \left(\delta_{F}\right)}{I_{z}} & \frac{b \bar{\beta}_{R}}{I_{z}} \\
-\frac{\bar{\beta}_{F} \cos \left(\delta_{F}\right)}{u m} & -\frac{\bar{\beta}_{R}}{u m}
\end{array}\right]
$$

Matrix $B_{1}$ is well defined, provided that vehicle velocity is non-null, which is always assumed in path tracking applications.

Cornering stiffnesses $C_{F}$ and $C_{R}$ are considered as the control variables, and a control law has to be designed to force $X_{1}$ to converge with $\bar{X}=[\dot{\bar{\theta}} \bar{\beta}]^{T}$, where $\dot{\bar{\theta}}$ is the measured yaw rate and $\bar{\beta}$ is given by (15). This latter variable can be seen as a consistent objective for $\hat{\beta}_{1}$, especially in a steady state. In transient phases, the priority put on the measured yaw rate (via observer gain tuning) limits the influence of the delays attached to $\bar{\beta}$. Convergence of $X_{1}$ to $\bar{X}$ can easily be obtained by choosing :

$$
u=B_{1}^{-1}\left[G_{1} \epsilon_{X}+\dot{\bar{X}}-A X_{1}\right]
$$

with $\epsilon_{X}=X_{1}-\bar{X}$ and $G_{1}$ a positive definite matrix, since injecting (18) into (16) leads to:

$$
\dot{\epsilon}_{X}=G_{1} \epsilon_{X}
$$

The only difficulty concerns the inversion of matrix $B_{1}$ required in (18). As can be observed in (17), $B_{1}$ is singular when sideslip angles are null, and badly conditioned when sideslip angles are close to 0 . Such cases occur when mobile robots move in a straight line on even ground, which is quite a standard situation. Therefore, in the sequel, the conditioning of matrix $B_{1}$ is tested prior to activating cornering stiffness estimation: if $B_{1}$ is badly conditioned (i.e. the robot does not turn), the previous cornering stiffness values are used as input values for dynamic sideslip angle observation (this is not a serious concern, since sliding is very limited in such situations). Cornering stiffness observation is turned on again when the system is excited sufficiently to allow relevant estimation. 


\subsection{Sideslip angle estimation}

Since relevant values of $C_{R}$ and $C_{F}$ are supplied on-line by the observer (18), all the parameters in the dynamic model (14) are now known. Standard observer theory can then be applied to this model in order to estimate sideslip angles. Since the dynamic effects are described in the model (14), it is expected that this second sideslip angle observer will display higher reactivity than the observer (5), previously built from extended kinematic model (1).

Let us first inject the third and fourth equations in (14) into the first two. The dynamic model (14) can then be presented as a linear state space form, with $X_{2}=\left[\begin{array}{ll}\dot{\theta} & \beta\end{array}\right]^{T}$ as state vector and $\delta_{F}$ as control variable:

$$
\dot{X}_{2}=A_{2} X_{2}+B_{2} \delta_{F}
$$

where:

$$
A_{2}=\left[\begin{array}{cc}
\frac{-a^{2} C_{F}-b^{2} C_{R}}{u I_{z}} & \frac{-a C_{F}+b C_{R}}{I_{z}} \\
-\frac{a C_{F}-b C_{R}}{u^{2} m}-1 & -\frac{C_{F}+C_{R}}{u m}
\end{array}\right], \quad B_{2}=\left[\begin{array}{c}
\frac{a C_{F}}{I_{z}} \\
\frac{C_{F}}{u m}
\end{array}\right]
$$

(it has also been assumed that $\cos \delta_{F} \approx 1$ )

The standard observer equation associated with model (20) is:

$$
\dot{\hat{X}}_{2}=A_{2} \hat{X}_{2}+B_{2} \delta_{F}+G_{2} \tilde{X}_{2}
$$

where $\hat{X}_{2}=\left[\begin{array}{ll}\dot{\hat{\theta}}_{2} & \hat{\beta}_{2}\end{array}\right]^{T}$ is the observed state, $\bar{X}=\left[\begin{array}{ll}\dot{\bar{\theta}} & \bar{\beta}\end{array}\right]^{T}$ is the measured state (measured yaw rate and preliminary vehicle sideslip angle estimation (15)) and $\tilde{X}_{2}=\hat{X}_{2}-\bar{X}$ is the observer error. From (20) and (22), it can be deduced that:

$$
\dot{\tilde{X}}_{2}=\left(A_{2}+G_{2}\right) \tilde{X}_{2}
$$

Convergence of the observer error $\tilde{X}_{2}$ to zero is clearly achieved, provided that $G_{2}$ is chosen such that $A_{2}+G_{2}$ is negative definite. Of course, the settling time of the cornering stiffness observer (18) has to be set shorter than the settling time of this observer, in order that relevant values for $C_{R}$ and $C_{F}$ may be available in the $A_{2}$ and $B_{2}$ matrices. 
Once more, $\bar{\beta}$ has been chosen as the measurement associated with the second observed state, since its steady state value is always correct. In order to avoid misestimation of sideslip angles at transient phases, priority has again to be given (when tuning $G_{2}$ ) to the

convergence of $\dot{\hat{\theta}}_{2}$ (whose associated measurement is reliable) with respect to the convergence of $\hat{\beta}_{2}$, such that the proposed observer essentially relies on the dynamic model (14).

Finally, the front and rear tire sideslip angles to be used in the control law (10) can be obtained by injecting $\hat{\beta}_{2}$ into the third and fourth equations in (14):

$$
\left\{\begin{array}{l}
\hat{\beta}_{R}=\hat{\beta}_{2}-\frac{b \dot{\hat{\theta}}}{u} \\
\hat{\beta}_{F}=\hat{\beta}_{2}+\frac{a \dot{\hat{\theta}}}{u}-\delta_{F}
\end{array}\right.
$$

The equations (24) constitute the mixed kinematic and dynamic sideslip angle observer. As demonstrated below, when off-road mobile robots move at high speed, observer (24) permits, with respect to the observer (5), improvement in the robustness and reactivity of sideslip angle estimation, and therefore the performances of path tracking.

\section{Advanced simulation results}

The capabilities of the mixed kinematic and dynamic sideslip angle observer (24) were first investigated through simulations, in order that the same conditions could be reproduced in several runs with different settings. Simulations were carried out using Adams multibody dynamic simulation software coupled with MatLab/Simulink software.

\subsection{Simulation description}

\subsubsection{Mobile robot definition}

A virtual mobile robot was designed with the celebrated Adams simulation software.

Its properties were chosen to match the features of the actual experimental device to be presented in section 5. This robot, depicted in Figure 7, is composed of a suspended chassis 


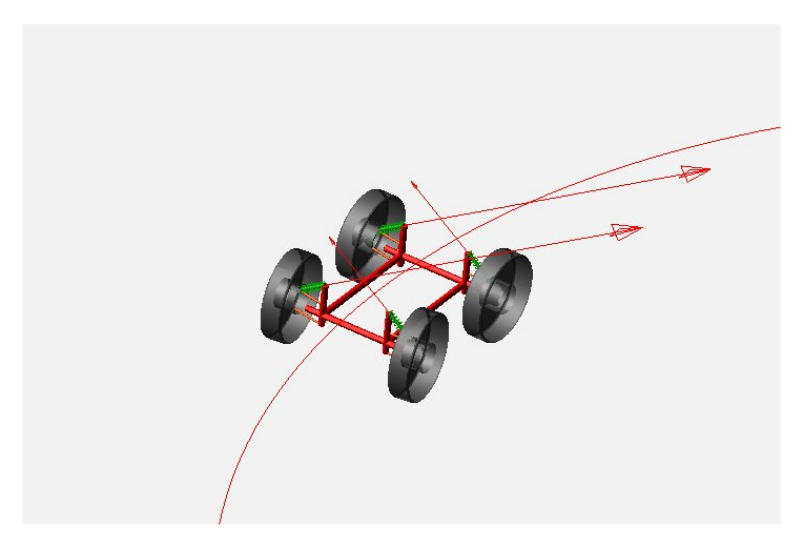

Figure 7: Simulated robot

attached to four wheels. The two front ones are steerable, while the rear ones are motorized and then impose robot velocity (to be precise, it is their angular acceleration that is actually controlled). The geometric and inertial parameters of this virtual robot (which can easily be modified) are:

$\begin{array}{cc}\text { Chassis mass }(\mathrm{m}) & 300 \mathrm{~kg} \\ \text { Wheel mass } & 17 \mathrm{~kg} \\ \text { Yaw inertia }\left(I_{z}\right) & 270 \mathrm{~kg} \cdot \mathrm{m}^{2} \\ \text { Wheelbase }(L) & 1.2 \mathrm{~m} \\ \text { Rear half-wheelbase }(b) & 0.6 \mathrm{~m} \\ \text { Track } & 1.3 \mathrm{~m}\end{array}$

Table 1: Simulated robot parameters

\subsubsection{Path tracking scenario}

The reference path, see Figure 8, is composed of a short straight line, continued with a long curve of constant curvature. The connexion between these two parts consists of a clothoid curve, in order to ensure curvature continuity and therefore path kinematic admissibility. The radius of curvature of the circular part of the reference path is $8 \mathrm{~m}$. In view of the robot's geometric features, the expected steering angle, if motion without sliding could be 
achieved, would be $8.5^{\circ}$.

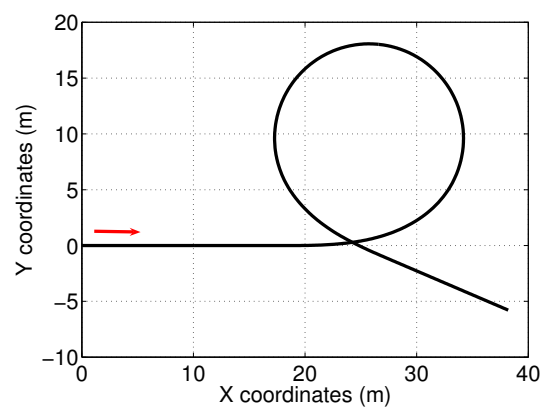

Figure 8: Path to be followed in simulation

Several path tracking simulations have been run, considering two vehicle velocities $(v=$ $4 m . s^{-1}$ and $v=8 m . s^{-1}$ ) and the following three control strategies:

- CWoS - Control law without sliding compensation. Control law (10) is used, but sliding is neglected $\left(\beta_{F}\right.$ and $\beta_{R}$ are set to zero). The resulting control law could also have been derived from a classical Ackermann model. In the following figures, the results related to CWoS are depicted with a black plain line.

- CWSKinObs - Control law with sideslip angles estimated via the extended kinematic observer (5). In the following figures, the simulation results related to CWSKinObs are depicted with a red dotted line.

- CWSDynObs - Control law with sideslip angles estimated via the mixed kinematic and dynamic observer (24). In the following figures, the simulation results related to CWSDynObs are depicted with a magenta dashed line.

For each test, the mobile robot starts $50 \mathrm{~cm}$ away from the reference path. 


\subsubsection{Grip conditions}

The grip conditions were entered via the contact interface of the Adams software, which allows specification of general non-linear behaviors at the tire/ground contact area. Terrain with low adherence was investigated. For the simulation runs carried out with $v=4 \mathrm{~m}_{\mathrm{s}} \mathrm{s}^{-1}$, the contact parameters are representative of a wet grass terrain. If the contact conditions are kept unchanged when $v$ is set to $8 \mathrm{~m} . \mathrm{s}^{-1}$, then the simulated robot spins round during the curved part of the reference path shown in Figure 8. Slightly more adherent ground was therefore considered for the simulation runs carried out with $v=8 \mathrm{~m}^{-1} \mathrm{~s}^{-1}$ in order to ensure the controlability. The parameters entered in the Adams Coulomb Friction model in both cases are listed in table 2 and the cornering stiffnesses mentionned are issued from the contact parameters entered into Adams for each simulation case.

$\begin{array}{ccc}\text { Parameter name } & \text { Value at } 4 \mathrm{~m} . \mathrm{s}^{-1} & \text { Value at } 8 \mathrm{~m} . \mathrm{s}^{-1} \\ { } } & 1 & 1 \\ \text { Dynamic Coefficient } & 0.95 & 0.95 \\ \text { Stiction transition velocity } & 1500 \mathrm{~mm} \cdot \mathrm{s}^{-1} & 500 \mathrm{~mm} \cdot \mathrm{s}^{-1} \\ \text { Friction transition velocity } & 4000 \mathrm{~mm} \cdot \mathrm{s}^{-1} & 1500 \mathrm{~mm} \cdot \mathrm{s}^{-1} \\ \text { Equivalent cornering stiffness } & 8000 \mathrm{~N} / \mathrm{rad} & 40000 \mathrm{~N} / \mathrm{rad} \\ \text { (i.e. value during steady state phases) } & & \end{array}$

Table 2: Simulated grip condition parameters

\subsubsection{Control and observer settings}

The control and observer settings used in the simulation runs, as well as in the experiments to be described in forthcoming section 5 , are listed in table 3 .

Control gains $\left(K_{d}, K_{p}\right)$ are used to specify the desired settling distance of the path-following control law (6), i.e. the distance (evaluated along the reference path) to be covered by the vehicle to converge to the reference path from any starting configuration (see (Lenain et al., 


$$
\begin{array}{lll} 
& v=4 m . s^{-1}: & v=8 m . s^{-1}: \\
\text { Control gains } & K_{d}=0.3 & K_{d}=0.15 \\
& K_{p}=0.0225 & K_{p}=0.0056
\end{array}
$$

Horizon of prediction $H$

Preliminary sideslip angle observer $(G)$

Cornering Stiffness observer $\left(G_{1}\right)$

Dynamic sideslip angle observer $\left(G_{2}\right)$

\section{$0.8 s$}

$\left[\begin{array}{cc}10 & 0 \\ 0 & 5\end{array}\right]$

$\left[\begin{array}{cc}5 & 0 \\ 0 & 0.5\end{array}\right]$

$\left[\begin{array}{cc}0.5 & 0 \\ 0 & 0.05\end{array}\right]$

Table 3: Control and observer settings

2006)). Imposing a settling distance rather than a settling time is advantageous at low speed: from any given initial conditions, the vehicle's trajectory towards the reference path is then identical, whatever its velocity, and even if its velocity varies. In contrast, at high speed, actuator limitations can quickly be faced when trying to satisfy some given settling distance. Control law performance must then be specified as a settling time, to be chosen consistently with respect to the steering actuator dynamics. Since the pure delay and the settling time of the actuation device of our experimental platform are respectively $0.1 s$ and $0.8 s$, the settling time of the control law (6) was set to $5 s$. Consequently, $\left(K_{d}, K_{p}\right)$ were tuned, see table 3 , in order to impose respectively $20 \mathrm{~m}$ and $40 \mathrm{~m}$ settling distances when $v=4 m \cdot s^{-1}$ and $v=8 m \cdot s^{-1}$.

In contrast, the observer settings can all be straightforwardly interpreted as settling times (see (5), (19) and (23)): their tuning can then be specified independently of the vehicle's velocity. The chosen matrices $G_{1}$ and $G_{2}$, listed in table 3 , are consistent with the requirements introduced in Section 3.2: on the one hand, in both matrices, the gains associated with the observed yaw rate are ten times higher than the gains associated with the observed 
vehicle sideslip angle. This permits greater confidence to be placed in the reliable yaw rate measurement than in the inevitably delayed vehicle sideslip angle supplied by the preliminary kinematic observer (5). On the other hand, the gains of the cornering stiffness observer (i.e. matrix $G_{1}$ ) are ten times higher than those of the dynamic sideslip angle observer (i.e. matrix $G_{2}$ ). The settling time of the former observer is then much shorter than the settling time of the latter one, which is very much to be expected, since the latter observer depends on the values supplied by the former.

\subsection{Results with respect to path tracking accuracy}

Path tracking relying on the three control strategies was first performed at a speed of $4 \mathrm{~m} . \mathrm{s}^{-1}$ (path tracking errors are compared in Figure 9(a)), and then at a speed of $8 m . s^{-1}$ (tracking errors are reported in Figure 9(b)).

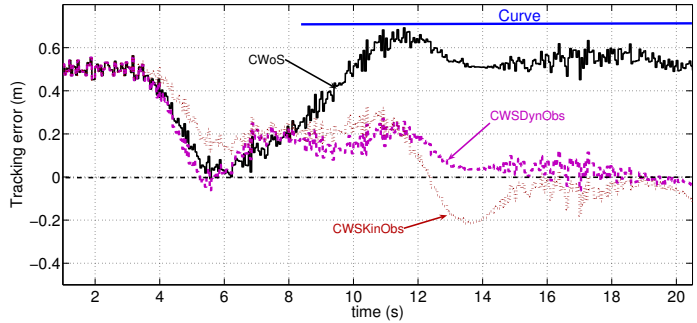

(a) Tracking errors at $4 m \cdot s^{-1}$

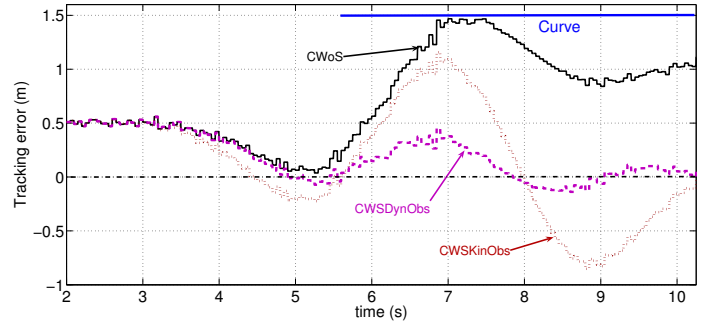

(b) Tracking errors at $8 m \cdot s^{-1}$

Figure 9: Comparison of tracking errors at different speeds

The importance of taking sliding into account in path tracking algorithms devoted to offroad mobile robots moving at high speed (and performing ample maneuvers) can easily be demonstrated: the tracking error resulting from the CWoS control algorithm is far from negligible, reaching a $50 \mathrm{~cm}$ steady value when the robot's velocity is $4 \mathrm{~m} . \mathrm{s}^{-1}$, and stabilizing around $1 m$ when it is $8 m . s^{-1}$. Such errors are unsatisfactory and may be dangerous, depending on the application considered. In contrast, the two control strategies accounting for sliding effects (CWSKinObs and CWSDynObs) display limited lateral deviation, despite sliding phenomena.

Nevertheless, whatever the vehicle velocity, overall path tracking performance is clearly more 
attractive when sideslip angles are estimated with the mixed kinematic and dynamic observer (24). When $v=4 \mathrm{~m} . \mathrm{s}^{-1}$, the lateral deviation converges asymptotically to small values in the curved part of the reference path with both control strategies, but CWSDynObs ensures faster convergence (lateral deviation returns to values less than $10 \mathrm{~cm}$ from $t=13 \mathrm{~s}$, compared to $t=15 \mathrm{~s}$ with CWSKinObs) with no negative overshoot (while a $20 \mathrm{~cm}$ negative overshoot is recorded with CWSKinObs). The differences between the two control strategies are accentuated when robot speed is increased. When $v=8 \mathrm{~m} . \mathrm{s}^{-1}$, the delay in the estimation of sideslip angles with the preliminary kinematic observer (5) significantly perturbs the robot's trajectory: when relying on CWSKinObs, a $1 \mathrm{~m}$ overshoot is recorded when the robot enters the curve, and it then follows the reference trajectory with quite large oscillations. In contrast, when relying on CWSDynObs, both the initial overshoot and the oscillations are satisfactorily reduced, so that the robot can quickly return close to the reference path (the lateral deviation values are inferior to $10 \mathrm{~cm}$ beyond $t=8 \mathrm{~s}$ ) despite low grip conditions.

\subsection{Results on cornering stiffness and sideslip angle estimation}

Such an improvement clearly follows from an increased reactivity of sideslip angle estimation, as can be observed in Figure 10: in the simulation run with $v=8 \mathrm{~m} \cdot \mathrm{s}^{-1}$ and CWSDynObs control strategy, the front and rear tire sideslip angles supplied by the preliminary kinematic observer (5) and the mixed kinematic and dynamic observer (24) were both recorded. Figure 10 shows that the latter observer is able, at transient phases (beginning/end of the curve) to describe sideslip angle variations reactively without adding any noise. The "actual" rear tire sideslip angle reconstructed from Adams software internal variables, shown in Figure 10(b), clearly highlights the relevancy of the estimated value supplied by observer (24). Of course, in a steady state, both observers supply approximately the same signal, as expected.

The capabilities of the cornering stiffness observer (18), used in CWSDynObs control strat- 


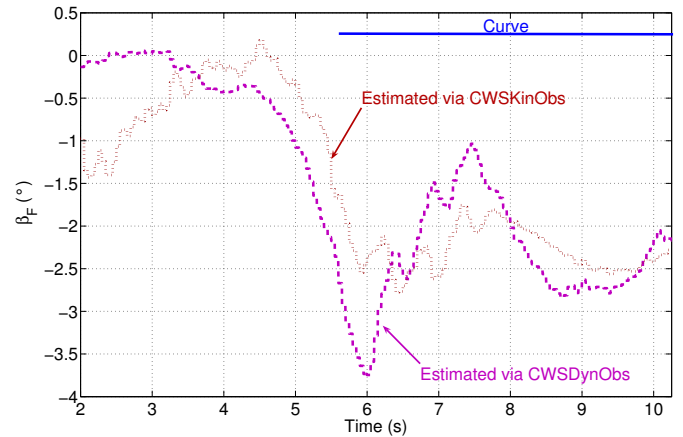

(a) Front tire sideslip angle

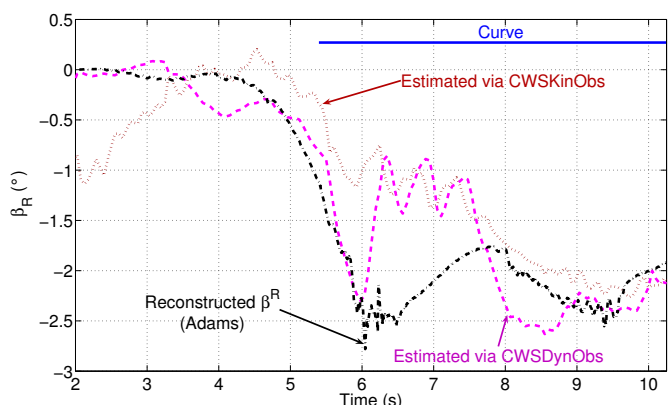

(b) Rear tire sideslip angle

Figure 10: Comparison of sideslip angle estimations

egy, are more specifically investigated in Figure 11: the front and rear cornering stiffnesses delivered by observer (18) in the previous simulation (CWSDynObs control strategy with $\left.v=8 m . s^{-1}\right)$ are reported, as well as the "actual" rear cornering stiffness reconstructed from Adams software internal variables. As long as the robot moves in a straight line (i.e. until $t=5.5 \mathrm{~s}$ ), the cornering stiffness adaptation is frozen, since matrix $B_{1}$ is badly conditioned: $C_{R}$ and $C_{F}$ are then equal to the chosen initial value $50000 \mathrm{~N} / \mathrm{rad}$. When the robot enters the curve, the adaptation becomes effective and estimates grip conditions quickly and realistically: after a transient phase when the steering actuator acts to bring the robot back to the reference path, $C_{R}$ and $C_{F}$ tend to almost constant values, as expected since the curvature of the reference path is constant. Their order of magnitude is then quite correct, in view of the rear cornering stiffness reconstructed from Adams data. Although the harsh grip conditions in this sharp curve correspond to the non-linear part of the tire/ground contact model, the observer (18) delivers relevant cornering stiffness values, and in turn enables accurate sideslip angle estimation and accurate path tracking.

\subsection{Sensitivity to dynamic parameters}

The mixed kinematic and dynamic observer (24) brings significant improvements in sideslip angle estimation, and consequently in path tracking accuracy, when off-road mobile robots move at high speed. Nevertheless, with respect to the extended kinematic observer (5), additional parameters are required: the longitudinal position of the center of gravity ( $a$ and 


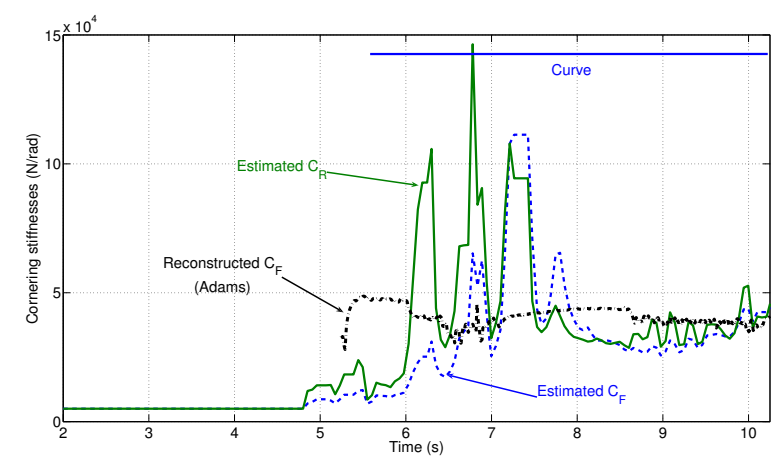

Figure 11: Adaptation of cornering stiffnesses

$b$ ), the robot mass $m$ and its inertia $I_{z}$ with respect to the vertical axis. Parameters $a, b$ and $m$ can easily be measured, but this is not the case for $I_{z}$. In order to investigate the sensitivity of the proposed approach to poorly known dynamic parameters, simulations with $v=8 \mathrm{~m} \cdot \mathrm{s}^{-1}$ were run injecting an erroneous mass $m=500 \mathrm{~kg}$ and an erroneous inertia $I_{z}=200 \mathrm{~kg} \cdot \mathrm{m}^{2}$ in CWSDynObs (their exact values, as well as the other parameter values are listed in table 1). Figure 12(a) compares the lateral deviation recorded with these wrong parameters to the tracking error obtained in the ideal case (i.e. when correct parameter values are used), and Figure 12(b) presents the influence of wrong parameters on the adapted cornering stiffnesses.

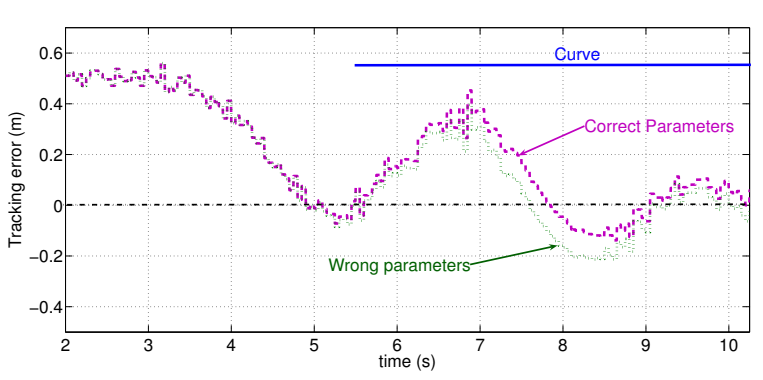

(a) Tracking error

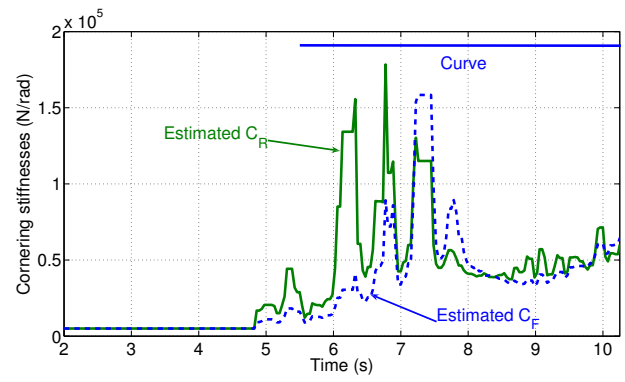

(b) Cornering stiffness modifications

Figure 12: Results with wrong dynamic parameters

It can be observed that the tracking error is not significantly modified when wrong mass and inertia are introduced. It was also demonstrable that sideslip angle estimation is unaltered too. In fact, path tracking accuracy is preserved thanks to the adaptation of cornering stiffnesses: it can be observed that cornering stiffness values in Figure 12(b) (with wrong 
dynamic parameters) and in Figure 11 (with correct dynamic parameters) are not identical in the transient phase (from $t=5 \mathrm{~s}$ to $t=8 \mathrm{~s}$ ), as in steady state (beyond $t=8 \mathrm{~s}$, cornering stiffness values in both figures differ by around $10000 \mathrm{~N} / \mathrm{rad}$ ). Thanks to these differences, sideslip angles can be equivalent in both simulation runs. Observer (24) appears therefore insensitive to erroneous inertial parameters. The same result holds for geometric parameters: path tracking accuracy is preserved if small errors (a few centimeters) are introduced into parameters $a$ and $b$. Nevertheless, larger errors in these parameters (more than $15 \mathrm{~cm}$ ) lead to a deterioration in path tracking accuracy: a $25 \mathrm{~cm}$ error on half-wheelbases leads to a $20 \mathrm{~cm}$ steady lateral deviation at the speed of $8 \mathrm{~m} \cdot \mathrm{s}^{-1}$ under consideration.

\section{$5 \quad$ Experimental results}

\subsection{Experimental mobile platform}

The experimental platform is shown in Figure 13. It consists of an electric off-road vehicle, whose weight and maximum speed are respectively $350 \mathrm{~kg}$ and currently $5 \mathrm{~m} . \mathrm{s}^{-1}$. This vehicle can climb slopes up to $45^{\circ}$.

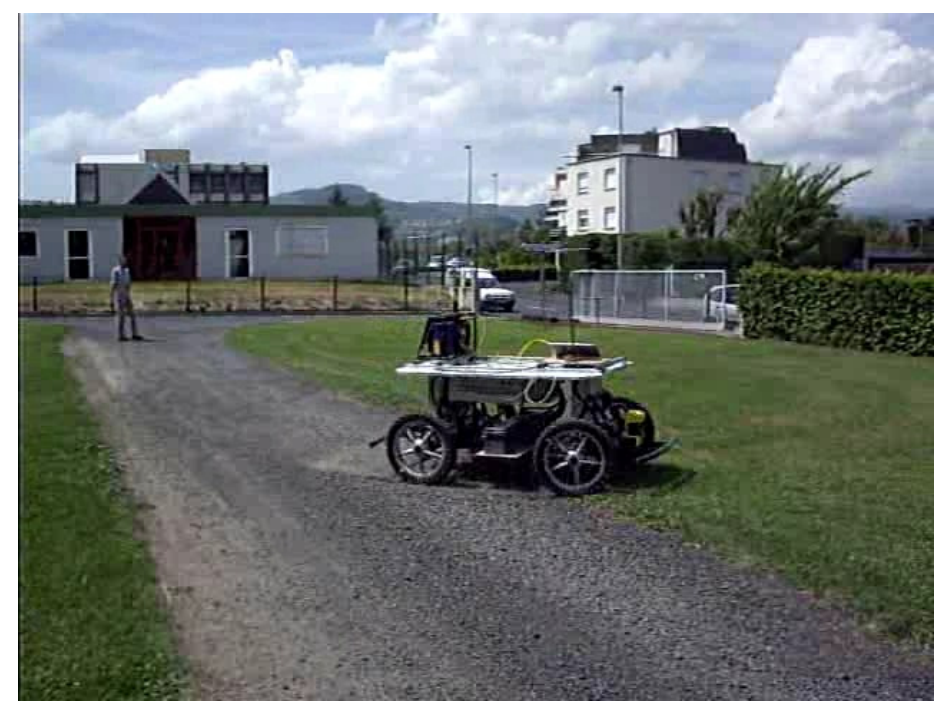

Figure 13: Experimental platform

The main exteroceptive sensor on board is a Dassault-Sercel dual frequency "Aquarius 5002" 
RTK-GPS receiver, which can supply an absolute position accurate to within $2 \mathrm{~cm}$, at a $10 \mathrm{~Hz}$ sampling frequency. The GPS antenna is located vertically above the center of the rear axle, so that the absolute position of point $R$ (i.e. the point to be controlled, see Figures 1 and 4 ) is straightfowardly obtained from the sensor. The vehicle heading is then inferred via a Kalman filter. In addition, a gyrometer supplying a yaw rate measurement accurate to within $0.1^{\circ} . s^{-1}$ is fixed on the chassis, to provide the cornering stiffness observer (18) and dynamic sideslip angle observer (24) with this information.

The data supplied by these sensors are sent via an RS232 serial communication port to a computer, where they are processed with a dedicated software framework managing unsynchronized sensors and delayed observations (see (Tessier et al., 2006)) and then considered by the observer and control algorithms implemented in high level language $(\mathrm{C}++)$. The front wheel steering angle delivered by these algorithms is then sent to an inner closed loop driving the steering actuator (the actual front wheel angle is measured via an absolute encoder). The steady state error of the inner closed loop is less than $0.1^{\circ}$. Its initial delay and settling time are respectively equal to 0.1 second and 0.8 second.

Finally, preliminary measurements and calibrations have supplied the following dynamic parameter set for this robot, to be reported in the observer algorithms:

$\begin{array}{cc}\text { Total mass } & m=350 \mathrm{~kg} \\ \text { Yaw inertia } & I_{z}=270 \mathrm{~kg} \cdot \mathrm{m}^{2} \\ \text { Wheelbase } & L=1.2 \mathrm{~m} \\ \text { Rear half-wheelbase } & b=0.58 \mathrm{~m}\end{array}$

Table 4: Experimental robot dynamic parameters

\subsection{Description of the experiments}

First of all, with the experimental platform described above, a reference trajectory was manually recorded: once more, it is composed of a straight line connected smoothly to a 
curve with a constant $4.5 \mathrm{~m}$ radius of curvature, see Figure 14. The robot steering angle in the circular part of the path is close to $\delta_{F}=15^{\circ}$.

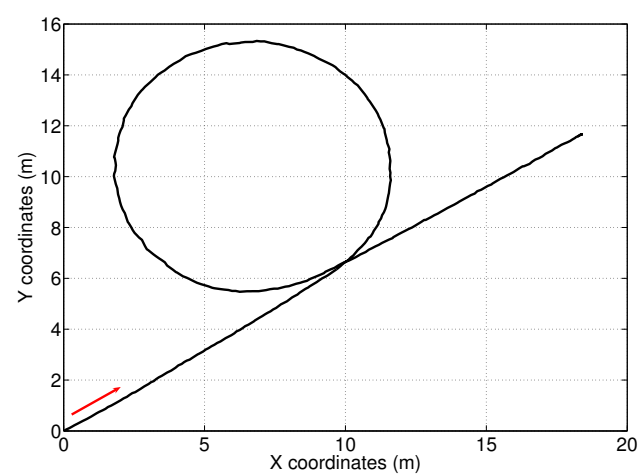

Figure 14: Reference trajectory manually recorded

Path tracking experiments were performed at $v=3 m \cdot s^{-1}$ and $v=4 m \cdot s^{-1}$. The three control strategies described in section 4.1.2 were considered successively. The color code associated with each strategy in the forthcoming experimental results is identical to the one introduced in section 4.1.2. The control and observer settings are those used in the simulations where $v=4 \mathrm{~m} \cdot \mathrm{s}^{-1}$, listed on table 3 . A video-sequence showing a trial at $4 \mathrm{~m} \cdot \mathrm{s}^{-1}$ is available on the following website (https://projetfast.cemagref.fr/avancees/essai-tests-en-boucle-ferme/suiviclermont-4ms/view).

\subsection{Path tracking results}

The tracking errors recorded during each experiment are compared in Figure 15. Once more, it can be observed that the tracking error resulting from CWoS (i.e. when low grip conditions are not taken into account) is not negligible: a $1 \mathrm{~m}$ overshoot and a $60 \mathrm{~cm}$ steady value when $v=3 \mathrm{~m} . \mathrm{s}^{-1}$, a $1.5 \mathrm{~m}$ overshoot and more than $60 \mathrm{~cm}$ when covering the curve at $v=4 \mathrm{~m} \cdot \mathrm{s}^{-1}$. These results differ to some degree from those obtained in the simulation (Figure 9), since the actual grip conditions could not obviously be reproduced accurately and the radius of curvature of the reference path is smaller here. 


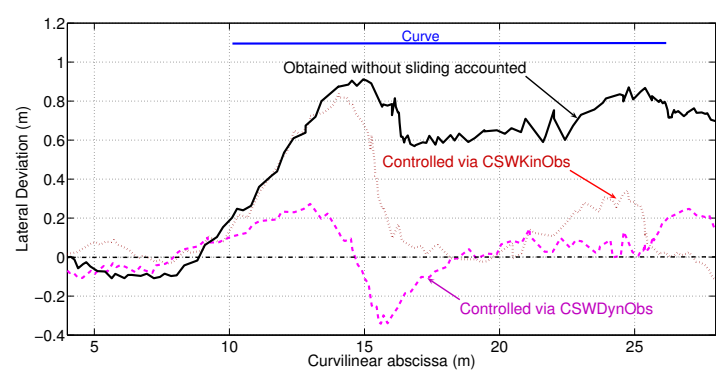

(a) Tracking errors at $3 m \cdot s^{-1}$

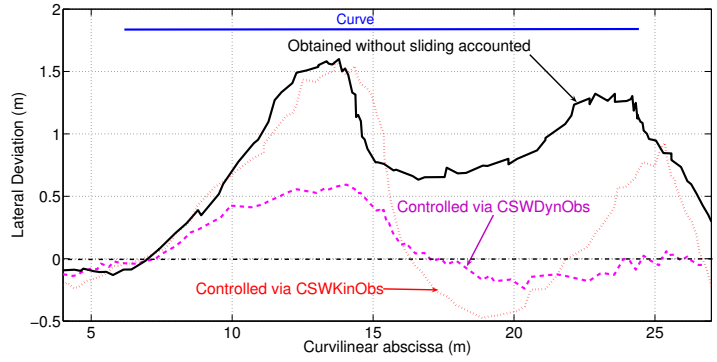

(b) Tracking errors at $4 m \cdot s^{-1}$

Figure 15: Comparison of tracking errors with different control strategies at different speeds

Path tracking performances are, of course, significantly improved when sliding effects are taken into account, and just as in simulation section 4 , it can be verified that the more attractive performances are obtained when the sideslip angles are estimated with the mixed kinematic and dynamic observer (24). When $v=3 \mathrm{~m} \cdot \mathrm{s}^{-1}$ (Figure 15(a)), both CWSKinObs and CWSDynObs control strategies ensure asymptotically small lateral deviations in the curved part of the reference path. However, on the one hand, the overshoot observed when relying on CWSDynObs is significantly smaller $(30 \mathrm{~cm}$ against $80 \mathrm{~cm}$ with $\mathbf{C W S K i -}$ nObs), and on the other hand the tracking accuracy when describing the curve is more satisfactory with CWSDynObs: the lateral deviation values stay under $10 \mathrm{~cm}$ from $s=18$ $m$ to $s=25 \mathrm{~m}$, when the robot moves away from the reference trajectory by $30 \mathrm{~cm}$ with CWSKinObs (at $s=23 \mathrm{~m}$ ).

When $v=4 \mathrm{~m} \cdot \mathrm{s}^{-1}$ (Figure 15(b)), the CWSKinObs control strategy is no longer able to keep the robot tightly close to the reference path in the curved part: the delay in the estimation of the sideslip angles with the preliminary kinematic observer (5) leads to large oscillations, just like those already observed in simulation with $v=8 \mathrm{~m}^{-s^{-1}}$ (Figure $9(\mathrm{~b})$ ). In contrast, the CWSDynObs control strategy still enables satisfactory path tracking: the overshoot is limited to $55 \mathrm{~cm}$ and then the robot returns close to the reference path (the lateral deviation values stay under $15 \mathrm{~cm}$ beyond $s=16 \mathrm{~m}$ ), despite the difficult grip conditions and the small radius of curvature of the reference path. 


\subsection{Observer performance}

Figure 16(a) and 16(b) present respectively the front and rear tire sideslip angles supplied by the mixed kinematic and dynamic observer (24) and by the preliminary kinematic observer (5), recorded during the experiment with CWSDynObs control strategy and $v=4$ $m . s^{-1}$.

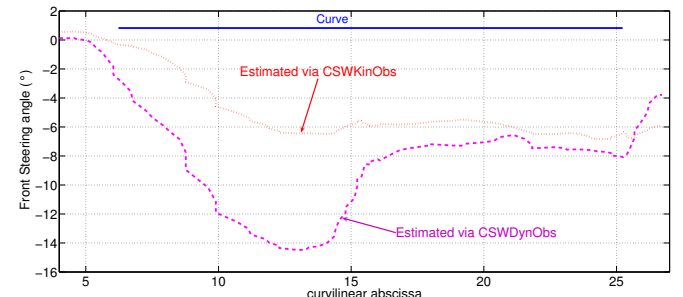

(a) Front tire sideslip angle

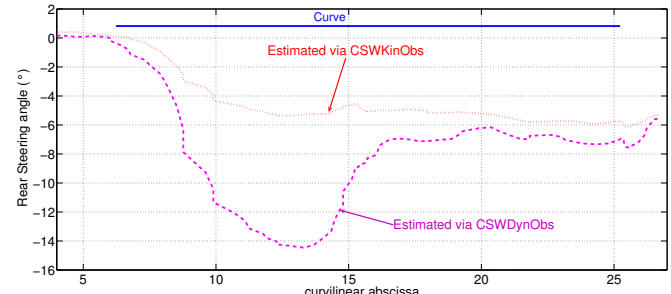

(b) Rear tire sideslip angle

Figure 16: Comparison of observed sideslip angles

It can be observed, just as in simulation section 4, that the mixed kinematic and dynamic observer (24) can follow sideslip angle variations reactively when the robot enters the curve. Since relevant sideslip angle values are available, the CWSDynObs control strategy can quickly compensate for sliding phenomena and can therefore ensure highly accurate path tracking, as verified in Figure 15(b). In contrast, the delay in the estimation of sideslip angles with the preliminary kinematic observer (5) leads, in these harsh conditions, to largely erroneous sideslip angle values for the first half of the curved part of the reference path. If these erroneous values were used in control law (6) (CWSKinObs control strategy), then sliding phenomena would no longer be properly compensated. Figure 15(b) has shown that the robot would then display oscillating behavior, which in turn reinforces sliding phenomena and consequently the negative impact of the delay in the sideslip angle estimation supplied by the observer (5), in a vicious circle. Finally, as Figure 16 shows, the higher reactivity presented by observer (24) is obtained without any increase in noise level on the sideslip angle signal (this is possible because a partial dynamic model is used), so that this signal can actually be sent to the control law (6). It contrasts with what is obtained when the gains of the observer (5) are increased in an attempt to improve its reactivity. 


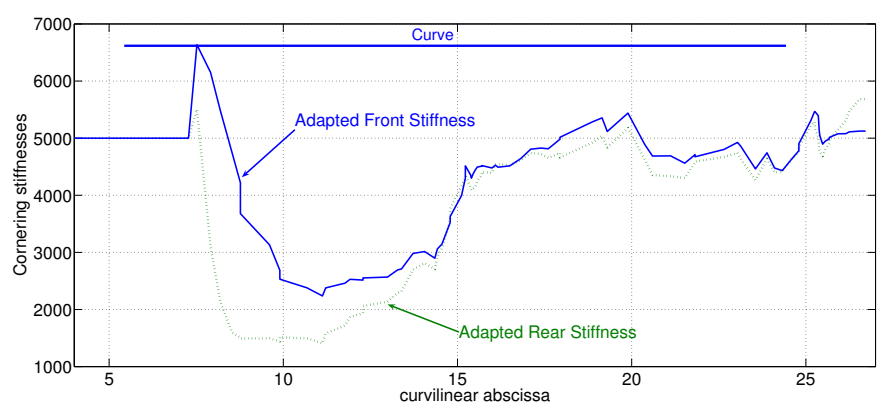

Figure 17: Adapted cornering stiffnesses

The cornering stiffnesses supplied by the observer (18) are reported in Figure 17. Once more, when the robot moves in a straight line (i.e. until $s=7 \mathrm{~m}$ ), the adapted stiffnesses are constant (the adaptation is frozen, as matrix $B_{1}$ defined in (17) is badly conditioned. $C_{R}$ and $C_{F}$ are then equal to the chosen initial value $5000 \mathrm{~N} / \mathrm{rad}$ ). As soon as the robot enters the curve, the adaptation turns active and can describe grip condition variations. As can be seen in Figure 17, the grip conditions are rather similar for both front and rear axles, as is to be expected since the center of gravity is close to the robot center, and they tend to almost constant values, since the curvature of the reference path is constant.

\section{Conclusion and future work}

This paper proposes a mixed kinematic and dynamic sideslip angle observer designed for high speed path tracking in an off-road environment. With respect to pure kinematic approaches, the use of a dynamic representation for the estimation of grip conditions improves reactivity in sliding variable adaptation and consequently in path tracking accuracy. This improvement appears to be significant at high speed, since any delay in sliding estimation leads to oscillating behavior. The kinematic part of the overall observer enables operation without a perfect knowledge of the vehicle's dynamic parameters (and especially tire/ground contact properties), which would have been unrealistic in practical applications.

Full scale experiments are reported. For the present, the maximum velocity of the exper- 
imental vehicle is $5 m . s^{-1}$. Nevertheless, even at this speed, when the robot has to track paths with small radii of curvature on ground where adherence is low, then the relevance of the proposed approach is demonstrable: significant improvements in robot behavior and in path tracking accuracy can be recorded. An upgrade of the experimental platform, allowing a maximum velocity of $8 \mathrm{~m} . \mathrm{s}^{-1}$ to be attained, is under development and will enable further investigation of the benefits and relevance of the approach, already indicated in advanced simulations carried out with Adams multibody simulation software.

Beyond the improvement in path tracking accuracy at high speed in an off-road context, one of the results derived from the proposed algorithms is that grip conditions can be accurately estimated on-line (through cornering stiffness adaptation). Such information is attractive, not only from a motion control point of view, but also with respect to stability preservation. As already pointed out in previous work, grip conditions directly impact the risk of rollover and overturn, which has obviously to be considered in fully autonomous applications, especially at high speed and in a natural environment. The developments proposed in this paper open the way to an integrated control algorithm, able to manage robot motion as well as its integrity, thus providing the robot with a high degree of autonomy.

\section{References}

Bakker, E., Nyborg, L., and Pacejka, H. (1987). Tyre modeling for use in vehicle dynamics studies. In International Congress of the Society of Automotive Engineers (SAE), pages 2190-2204, Warrendale, Pensylvania (USA).

Bouton, N., Lenain, R., Thuilot, B., and Martinet, P. (2008). A tire stiffness backstepping observer dedicated to all-terrain vehicle rollover prevention. Advanced Robotics, 22(12):1267-1285.

Bruinsma, J. (2003). World agriculture: towards 2015/2030. An FAO perspective. Technical report, Food and Agriculture Organisation.

Buehler, M., Iagnemma, K., and Sanjiv, S. (2007). The 2005 DARPA Grand Challenge: the 
great robot race. Springer Tracts in Advanced Robotics, 36.

Canudas de Wit, C., Olsson, H., Astrom, K., and Lischinsky, P. (1995). A new model for control of systems with friction. IEEE Transactions on Automatic Control, 40(3):419425.

Cariou, C., Lenain, R., Thuilot, B., and Martinet, P. (2008). Adaptive control of four-wheelsteering off-road mobile robots: Application to path tracking and heading control in presence of sliding. In IEEE/RSJ International Conference on Intelligent Robots and Systems (IROS), pages 1759-1764, Nice, France.

Eaton, R., Katupitiya, J., Siew, K. W., and Dang, K. S. (2008). Precision guidance of agricultural tractors for autonomous farming. In 2nd Annual IEEE Systems Conference, pages $1-8$.

Ellouze, M. and d'Andréa-Novel, B. (2000). Control of unicycle-type robots in the presence of sliding effects with only absolute longitudinal and yaw velocities measurement. European Journal of Control, 6(6):567-584.

Hao, F., Lenain, R., Thuilot, B., and Martinet, P. (2005). Robust adaptive control of automatic guidance of farm vehicles in the presence of sliding. In IEEE International Conference on Robotics and Automation (ICRA), pages 3113-3118, Barcelona (Spain).

Lenain, R., Thuilot, B., Cariou, C., and Martinet, P. (2006). High accuracy path tracking for vehicles in presence of sliding. Application to farm vehicle automatic guidance for agricultural tasks. Autonomous robots, 21(1):79-97.

Lenain, R., Thuilot, B., Cariou, C., and Martinet, P. (2007). Adaptive and predictive path tracking control for off-road mobile robots. European Journal of Control, 13(4):419-439.

Lucet, E., Grand, C., Salle, D., and Bidaud, P. (2008). Stabilization algorithm for a high speed car-like robot achieving steering maneuver. In IEEE International Conference on Robotics and Automation (ICRA), pages 2540-2545, Pasadena, California (USA).

Micaelli, A. and Samson, C. (1993). Trajectory tracking for unicycle-type and two-steeringwheels mobile robots. INRIA technical report, ( $\left.\mathrm{n}^{\circ} 2097\right)$. 
Msirdi, K. N., Jaballah, B., Naamane, A., and Messaoud, H. (2008). Robust observers and unknown input observers for estimation, diagnosis and control of vehicle dynamics. In IEEE/RSJ 2008 International Conference on Intelligent Robots and Systems (IROS), pages 48-57, Nice, France.

Richalet, J. (1993). Industrial applications of model based predictive control. Automatica, 29:1251-1574.

Ryu, J.-C., Agrawal, S., and Franch, J. (2008). Motion planning and control of a tractor with a steerable trailer using differential flatness. ASME Transactions, Journal of Computational and Nonlinear Dynamics, 3(3):031003.1-8.

Samson, C. (1995). Control of chained systems. application to path following and timevarying point stabilization of mobile robots. IEEE Transactions on Automatic Control, 40(1):64-77.

Schofield, B., Hägglund, T., and Rantzer, A. (2006). Vehicle dynamics control and controller allocation for rollover prevention. In IEEE International Conference on Control Applications, Munich, Germany.

Siegwart, R. and Nourbakhsh, I. R. (2004). Introduction to Autonomous Mobile Robots. MIT Press, 2004.

Tai, M., Hingwe, P., and Tomizuka, M. (2004). Modeling and control of steering system of heavy vehicles for automated highway systems. IEEE/ASME Transactions on Mechatronics, 9(4):609-618.

Tessier, C., Cariou, C., Debain, C., Chausse, F., Chapuis, R., and Rousset, C. (2006). A realtime, multi-sensor architecture for fusion of delayed observations: application to vehicle localization. Intelligent Trasnportation Systems Conference (ITSC), pages 1316-1321.

Unyelioglu, K., Hatipoglu, C., and Ozguner, U. (1997). Design and stability analysis of a lane following controller. IEEE Transactions on Control Systems Technology, 5(1):127-134.

Wang, D. and Low, C. (2007). An analysis of wheeled mobile robots in the presence of 
skidding and slipping: Control design perspective. In IEEE International Conference on Robotics and Automation (ICRA), pages 2379-2384, Roma, Italy. 